\title{
Immunohistochemical evaluation of bone metastases
}

\author{
Costantino Errani ${ }^{1}$, Andreas F. Mavrogenis ${ }^{2}$, Panayiotis D. Megaloikonomos ${ }^{2}$, \\ Thekla Antoniadou ${ }^{2}$, Diego Antonioli ${ }^{1}$, Sofia Avnet ${ }^{3}$, Andrea Pellacani $^{3}$, Nicola Baldini ${ }^{3}$
}

Introduction. Metastases are the most common type of malignancy involving the bone, while bone is the third most frequent site for metastases, after the lung and liver. In some patients, medical history, physical and laboratory examination are not conclusive to identify the primary tumor site. In such cases a bone biopsy and immunohistochemical analysis may contribute to the diagnosis, determination of appropriate treatment and evaluation of prognosis. In this study, we tried to evaluate the imunochistochemical expression in bone metastases.

Material and methods. We reviewed 125 patients, with a mean age of 63 years, treated for bone metastases in our institution. All patients received palliative orthopaedic surgery for bone metastatic carcinoma. Fifty-eight patients had already an established diagnosis of the primary tumor, while 67 patients presented metastases with an unknown primary tumor origin. Immunohistochemical analysis was performed to intra-operative bone biopsy specimens. The expression of cytokeratine 7, cytokeratin 20 and the expression of a panel of other organ-specific markers were recorded. In patients with a known primary tumor, we examined the relationship between the origin of metastases, as suggested by the cytokeratin phenotype, compared with the one indicated by the initial histological diagnosis. We also recorded the efficacy of organ-specific markers to identify the primary tumor origin in epithelial bone metastases and we evaluated the prognosis between patients with a immunohistologically determined primary tumor origin, with those with an undetermined one.

Results. Associations of cytokeratine 7 and cytokeratine 20 expression confirmed diagnosis in 51 out of the 58 patients (88\%) with a known primary tumor (Cohen's K test 0.79 SE $0.80, \mathrm{P}<0.0005$ ). Immunohistochemical analysis also contributed to establish the diagnosis of patients with an unknown primary tumor, yielding diagnosis in 35 out of the 67 cases (52\%). Patients with an immunochistologically undetermined primary tumor site presented a statistically significant poorer prognosis.

Conclusions. Cytokeratine 7 and cytokeratine20 are useful immunochistochemical markers in determining a preliminary evaluation of bone metastases. Organ-specific immunohistochemical markers have a reliable role in either suggesting or confirming the possible origin of metastases. An indeterminate immunohistochemical phenotype seems to relate to a less differentiated lesion, with a worse prognosis.

NOWOTWORY J Oncol 2017; 67, 1:1-6

Key words: bone metastases, immunohistochemistry, cytokeratine 7, cytokeratine 20, organ specific markers, cancer of unknown primary origin

\footnotetext{
${ }^{1}$ Department of Orthopaedics, Istituto Ortopedico Rizzoli, Bologna, Italy

${ }^{2}$ First Department of Orthopaedics, National and Kapodistrian University of Athens, School of Medicine, Athens, Greece ${ }^{3}$ Laboratory for Orthopaedic Pathophysiology and Regenerative Medicine, Istituto Ortopedico Rizzoli, Bologna, Italy
} 


\section{Introduction}

Metastasis is the most common type of malignancy involving the bone, whereas bone represents the third most frequent site for metastases, after the lung and liver [1-3]. Bony lesions may be the first manifestation of malignancy in $25-30 \%$ of cases, and sometimes the only manifestation; as in metastatic disease of unknown origin [1]. The number of cancer survivors has been rising in recent years. The 5-year survival rate in breast and colorectal cancer is reported to be more than $60 \%$ and $85 \%$, respectively. Accordingly, the 5 -year survival rate for patients with prostate cancer is reported to be more than $95 \%$. In this setting, the increase in the overall survival in cancer patients may result in an increase in the overall incidence of bone metastases $[4,5]$.

In general, previous medical history, physical examination, laboratory tests, and imaging modalities are usually sufficient to establish a diagnosis of bone metastases. Differential diagnosis includes primary malignant bone tumors (i.e. sarcoma or chondrosarcoma), hematologic diseases (i.e. multiple myeloma or lymphoma) and osteomyelitis. However, in cases of occult primary malignancies, diagnosis may be difficult and bone biopsy and histology may be necessary to determine the appropriate treatment and prognosis [1]. Determination of the type and origin of metastatic disease constitutes a challenging area in pathology. A microscopical evaluation may reveal specific morphological features suggestive of tumor lineage and origin. However, in the absence of distinctive characteristics, immunochistochemichal (IHC) evaluation may help in diagnosis. A panel of tissue- or organ-specific markers is typically used. Thus, many authors underline the important role that cytokeratines and other specific markers play in the discrimination of primary tumor lineage and determination of metastatic disease [6-13].

In this context, aiming to provide further insights concerning IHC marker expression in metastatic carcinomas, we reviewed bone-tissue biopsies in a series of patients with bone metastases. Our purpose was to evaluate the effectiveness of these specific markers in identifying (or verifying) the primary tumor site and determine if they can provide certain information concerning prognosis.

\section{Materials and methods}

We initially reviewed the medical files of 194 patients who were admitted and treated for bone metastases to our institution. All patients received palliative orthopaedic surgery. Pathology reports from intra-operative specimens of bone metastases were reviewed. 35 patients were excluded, because their primary tumor was other than carcinoma. Likewise, 34 patients were excluded because of incomplete clinical or pathological data in their files. A total of 125 patients ( 75 men and 50 women) were finally evaluated. The mean age of primary tumor diagnosis was 58.6 years (range, 33-86 years), while bone metastases
Table I. Anatomical distribution of bone metastases (in the case of multiple metastases, all sites were included; bone metastases in more than three sites were defined as diffuse)

\begin{tabular}{lc}
\hline Skeletal site & Patients $(\mathbf{n})$ \\
\hline Humerus & 32 \\
Radius & 5 \\
Femur & 40 \\
Tibia & 10 \\
Spine & 22 \\
Pelvis & 17 \\
Tarsus & 0 \\
Clavicle & 4 \\
Scapula & 7 \\
Diffuse & 5 \\
\hline
\end{tabular}

were diagnosed at a mean age of 63 years (range, 39-87 years). The time lapse from primary diagnosis of malignancy to the development of bone metastases was 40 months (range, 9-304 months). 51 patients (40.5\%) presented bone metastases at a point after the primary tumor diagnosis, while 74 patients (59.5\%) had already bone metastases at the time of initial diagnosis. Histological diagnosis of the primary tumor was already established at the time of the bone biopsy in 58 cases. In 84 patients (67\%), pain was the only clinical sign of metastases development. The mean time between onset of symptoms and metastases detection was 6 months (range, 1-50 months). 30 patients (24\%) presented a pathological fracture. The mean survival after bone metastasis detection was 14 months (range, 1-50 months).

An imaging work-up (plain radiographs, CT scans, MRI scans, bone scans) was reviewed to locate the sites of metastases, the nature of the lesions and their relationship with the adjacent tissues. Table I and Table II show the anatomical distribution of bone metastases and the primary tumor origin in our patients. Pathology reports of biopsies from bone metastases were reviewed in terms of IHC analysis. Tumor tissues were collected during surgical treatment of the bone lesion, and were then fixed for 2-5 days in neutral buffered formalin and then processed for decalcification protocol and for paraffin embedding. Fixed specimens were decalcified in $20 \%$ EDTA in $10 \mathrm{~N} \mathrm{NaOH}$ at pH 7.4 from several days up to several weeks with intermittent shaking to make a sure the solution was flowing around the bone. The decalcification time depended on the degree of mineralisation and the size of the specimen. Representative $5 \mu \mathrm{m}$ thick sections were mounted on a glass slide covered with $2 \%$ silane solution in acetone. After dewaxing in xylene and rehydration in ethanol, for standard histology, slides were stained with Hematoxylin \& Eosin staining. For immunoistochemistry, slides were processed for antigen retrieval by heating in 
Table II. A breakdown of the primary tumors in our patients with bone metastases

\begin{tabular}{lcc}
\hline Primary tumor & $\begin{array}{c}\text { Patients with bone } \\
\text { metastases }(\mathbf{n})\end{array}$ & Percentages \\
\hline Unknown & 67 & $53.6 \%$ \\
Lung & 5 & $4 \%$ \\
Kidney & 16 & $12.8 \%$ \\
Breast & 19 & $15.2 \%$ \\
Uterus & 3 & $2.4 \%$ \\
Esophagus & 2 & $1.6 \%$ \\
Liver & 2 & $1.6 \%$ \\
Colon Rectum & 1 & $0.8 \%$ \\
Stomach & 2 & $1.6 \%$ \\
Cervix & 1 & $0.8 \%$ \\
Prostate & 2 & $1.6 \%$ \\
Biliary tract & 1 & $0.8 \%$ \\
Small intestine & 1 & $0.8 \%$ \\
Bladder & 3 & $2.4 \%$ \\
Total & 125 & $100 \%$ \\
\hline
\end{tabular}

a microwave oven in $0.02 \mathrm{M}$ citrate buffer, pH 6.0. After cooling, tissue sections were incubated in $3 \%$ perhydrol solution to block the endogenous peroxidase reaction. Non-specific binding was blocked by incubation in $5 \%$ bovine serum albumin. Incubation with specific primary antibodies was then followed by incubation with a biotinylated secondary antibody. Sections were then covered with DAB and counterstained with Mayer's hematoxylin. Negative controls were also performed by omitting the primary antibody. The expression of cytokeratine 7 (CK7), the expression of cytokeratin 20 (CK20) and the expression of a panel of other organ-specific markers, according to the expected tissue/ /organ of origin, were recorded. CEA and CDX2 were examined for gastrointestinal adenocarcinoma, APS and FAP for prostate adenocarcinoma, ER, PGR, GCDFP for breast carcinoma, TTF1 for pulmonary adenocarcinoma, WT1 for renal adenocarcinoma, HEP for hepatic carcinoma, and TIR for thyroid adenocarcinoma.

In the patients with an already known primary tumor, we examined the relationship between the origin of metastases, as suggested by cytokeratin phenotype (CK7/CK20), compared with the one indicated by the initial histological diagnosis. Subsequently, we examined all the patients of the study evaluating the relationship between the origin of metastases, as suggested by the organ-specific IHC markers, and the one indicated by histological diagnosis. The expected and actual results obtained by $\mathrm{IHC}$ markers have been assessed by Cohen's K test, AUC ROC, sensitivity and specificity.

Statistical analysis was performed with SPSS v. 19. Comparison between the groups of patients was conducted with Pearson's Chi Square Test, calculated with the Monte Carlo method for small samples. Cohen's K test and ROC analysis were conducted to compare the estimated and effective values between the groups. The Kaplan-Meier survival analysis was performed, while the Breslow test was conducted to assess the different features of each group.

\section{Results}

The IHC results in bone metastasis specimens from hepatic carcinoma ( 2 patients; one identified by the phenotype $\mathrm{CK} 7+/ \mathrm{CK} 20+$ and the other by $\mathrm{CK} 7+/ \mathrm{CK} 20-)$ and small intestine carcinoma (1 patient; identified by the phenotype (K7-/CK20-) were not significant and were excluded. Hence, cytokeratin expression was evaluated in 55 cases out of the 58 cases with a known primary tumor in the Pearson's Chi Square test. Results of the cytokeratin phenotype in metastatic bone disease are shown in Table III; agreement between the cytokeratine phenotype and the primary histology of already diagnosed tumors is shown in Table IV. Results of Cohen's K test, AUC ROC, the sensitiv-

Table III. Cytokeratin phenotype expression in bone metastases specimens according to primary tumor sites. Patients with an unknown primary tumor site were excluded

\begin{tabular}{|c|c|c|c|c|c|}
\hline Metastases origin & Patients (n) & No. of cases responsive to CKs test & CK7 & CK20 & Significance \\
\hline Biliary tract & 1 & $1(100 \%)$ & + & + & \multirow{2}{*}{$P<0.005$} \\
\hline Bladder & 3 & $3(100 \%)$ & + & + & \\
\hline Breast & 19 & $18(95 \%)$ & + & - & \multirow{4}{*}{$P<0.005$} \\
\hline Uterus & 3 & $3(100 \%)$ & + & - & \\
\hline Esophagus & 2 & $2(100 \%)$ & + & - & \\
\hline Lung & 5 & $4(80 \%)$ & + & - & \\
\hline Stomach & 2 & $1(50 \%)$ & - & + & \multirow{2}{*}{$P=0.03$} \\
\hline Cervix & 1 & $1(100 \%)$ & - & + & \\
\hline Colon rectum & 1 & $1(100 \%)$ & - & + & \multirow{3}{*}{$P<0.005$} \\
\hline Prostate & 2 & $2(100 \%)$ & - & - & \\
\hline Kidney & 16 & $14(87.5 \%)$ & - & - & \\
\hline
\end{tabular}


Table IV. Relationship between primary histological results with cytokeratin expression in bone metastases. Patients with an unknown primary tumor site were excluded

\begin{tabular}{lcccc}
\hline $\begin{array}{l}\text { Agreement between primary histology \& cytokeratin } \\
\text { Phenotype in bone metastases }\end{array}$ & CK7+/CK20+ & CK7+/CK20- & CK7-/CK20+ & CK7-/CK20- \\
\hline Significant results & Biliary tract* & Lung* $^{*}$ & Colon - rectum & Kidney* $^{*}$ \\
& Bladder* & Breast $^{*}$ & Stomach $^{\circ}$ & Prostate $^{*}$ \\
& & Uterus* $^{*}$ & Cervix $^{\circ}$ & \\
Not significant results & Esophagus* & Breast \\
& Prostate & Kidney & Uterus \\
\hline
\end{tabular}

'Pearson's Chi Square test presented statistical significance $(P<0.05)$; ${ }^{\circ}$ Pearson's Chi Square test presented high statistical significance $(P<0.005)$

Table V. Results of Cohen's K test, AUC ROC, the sensitivity and specificity between expected and actual values of IHC (primary tumor and metastasis). Patients with an unknown primary tumor site were excluded

\begin{tabular}{lcccc}
\hline & CK 7+/CK 20+ & CK 7+/CK 20- & CK 7-/CK 20+ & CK 7-/CK 20- \\
\hline Sensibility & $100 \%$ & $93 \%$ & $100 \%$ & $80 \%$ \\
Specificity & $98 \%$ & $88 \%$ & $100 \%$ & $93 \%$ \\
Cohen's K & $0.85-$ SE 0.15* & $0.80-$ SE 0.08* & $1-$ SE 0.0 & $0.75-$ SE 0.96* \\
AUC ROC & $0.99 \pm 0.01^{*}$ & $0.90 \pm 0.05^{*}$ & $1 \pm 0.0^{*}$ & $0.87 \pm 0.06^{*}$ \\
\hline
\end{tabular}

${ }^{*} \mathrm{P}<0.001 ;{ }^{\circ} \mathrm{P}<0.05 ;{ }^{*} \mathrm{P}>0.05$

ity and specificity between expected and actual values of immunochistochemistry (primary tumor and metastases) are shown in Table V. Accordingly, patients with unknown primary tumor origin were excluded. The closer Cohen's K value was to 1 , the greater the probability that metastases came from the indicated site of the cytokeratin phenotype.
ICH verification was observed in 50 out of 58 patients (86.2\%) with known primary tumor origin. Cohen's K agreement between the primary tumor histology and the IHC results of bone metastases was 0.79 - SE $0.80, \mathrm{P}<0.0005$.

In 32 out of 67 patients (48\%) though, with unknown primary tumor origin, IHC identification was not possible; in

Table VI. Primary tumor origin and organ-specific IHC markers for epithelial metastases

\begin{tabular}{lccccccccc}
\hline Primary tumor & \multirow{2}{*}{$\begin{array}{c}\text { Number of } \\
\text { metastases }\end{array}$} & \multicolumn{6}{c}{ Diagnosis of tumor origin after organ-specific IHC markers test on bone metastases } \\
\cline { 3 - 10 } & & Unknown & Lung & Kidney & Breast & Liver & Rectum & Stomach & Prostate \\
\hline Unknown & 67 & 32 & 12 & 6 & 7 & - & 4 & 2 & 4 \\
Lung & 5 & 3 & 2 & - & - & - & - & - & - \\
Kidney & 16 & 2 & - & 14 & - & - & - & - & - \\
Breast & 19 & 2 & - & - & 17 & - & - & - & - \\
Uterus & 3 & 3 & - & - & - & - & - & - & - \\
Esophagus & 2 & 1 & 1 & - & - & - & - & - & - \\
Liver & 2 & - & - & - & - & 2 & - & - & - \\
Rectum & 1 & - & - & - & - & - & 1 & - & - \\
Stomach & 2 & - & - & - & 1 & - & - & 1 & - \\
Cervix & 1 & - & - & - & - & - & 1 & - & - \\
Prostate & 2 & - & - & - & - & - & - & - & 2 \\
Biliary Tract & 1 & 1 & - & - & - & - & - & - & - \\
Small Intestine & 1 & - & - & 1 & - & - & - & - & - \\
Bladder & 3 & 3 & - & - & - & - & - & - & - \\
TOTAL & 125 & 47 & 15 & 21 & 25 & 2 & 6 & 3 & 6 \\
\hline
\end{tabular}


Table VII. The table shows the survival rate of patients based on the IHC results in bone metastases (patients with lung, renal and breast primary carcinoma)

\begin{tabular}{|c|c|c|c|}
\hline \multirow{2}{*}{$\begin{array}{l}\text { Metastases site } \\
\text { of origin }\end{array}$} & \multirow{2}{*}{$\begin{array}{l}\text { Results with } \\
\text { IHC markers }\end{array}$} & \multicolumn{2}{|c|}{ Survival } \\
\hline & & $\begin{array}{l}\text { No. pts with } \\
\text { survival } \\
<12 \text { months }\end{array}$ & $\begin{array}{c}\text { No. pts with } \\
\text { survival } \\
>12 \text { months }\end{array}$ \\
\hline \multirow[t]{2}{*}{ Lung } & Undetermined & 3 & - \\
\hline & Determined & 1 & 1 \\
\hline \multirow[t]{2}{*}{ Kidney } & Undetermined & 1 & - \\
\hline & Determined & 4 & 11 \\
\hline \multirow[t]{2}{*}{ Breast } & Undetermined & 2 & 1 \\
\hline & Determined & 5 & 11 \\
\hline
\end{tabular}

the remaining 52\%, IHC identification was achieved (Tab. VI). A false negative $\mathrm{ICH}$ result was provided in 3 out of 5 cases (60\%) with lung carcinoma. Likewise, a false negative result was provided in 2 out of 16 patients (12.5\%) with renal carcinoma, and in 2 out of 19 patients with breast carcinoma (10.5\%). The majority of patients in this series presented lung, renal or breast carcinoma. Table VII shows the relationship between the 1-year survival of these patients and their corresponding IHC expression in bone metastases. It should be noticed that 3 out of 4 patients (75\%) with bone metastases from lung carcinoma that died within a year, had an undetermined IHC phenotype. On the other hand, only 4 out of 15 patients (26.6\%) with IHC determined metastatic renal carcinoma and 5 out of 16 patients (31.2\%) with determined metastatic breast carcinoma died within a year. Overall, patients with undetermined $\mathrm{IHC}$ results in bone metastases had a worse survival rate than those with determined results $(P=0.007$, Breslow test; Fig. 1). Gender, an unknown primary tumor location, and the presentation of a pathological fracture, were not found to be statistically significant prognostic factors in our series $(P>0.05$, Breslow test).

\section{Discussion}

Cytokeratins consist of a family of over 20 polypeptides. They are intermediate filament proteins in epithelial cells that may be used to identify epithelial differentiation, as their expression pattern is usually preserved in neoplastic cells $[11,14]$. Monoclonal antibodies against cytokeratin polypeptides have been established to evaluate their tissuespecific expression. Cytokeratins have been reported to be effective in distinguishing between various subsets of primary and metastatic carcinomas in different anatomical sites. More specifically, many authors have studied the coordinate expression of CK7 and CK20 and reported the high sensibility and specificity of their association [6-13]. In our study we found that when these markers are both positive, both negative or when CK7 is positive and CK20 is negative there is relative success in finding possible origins in finding

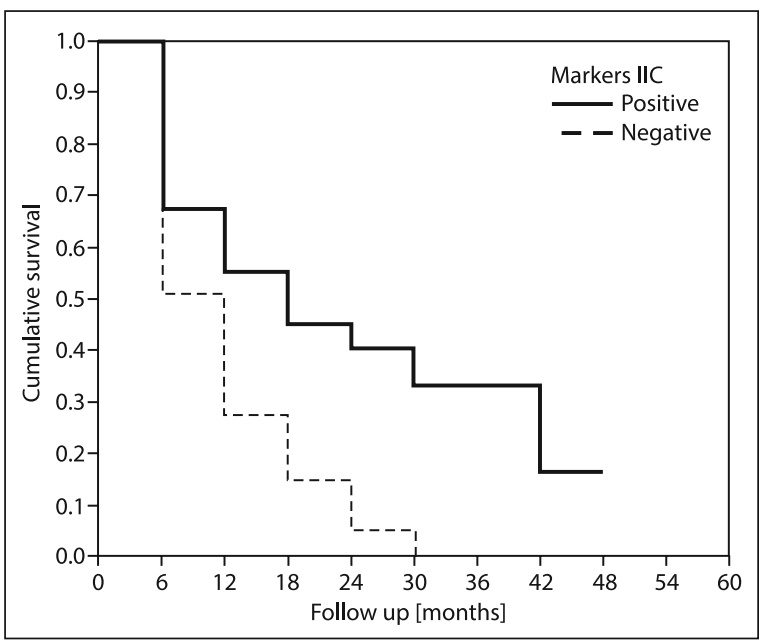

Figure 1. Patients with undetermined results in the $\mathrm{ICH}$ evaluation of bone metastases had a poorer 1-year survival rate than those with the determined phenotype ( $P=0.007$ at Breslow test)

possible origins for bone metastasis from carcinoma. Additionally, we observed that the combination of a negative CK7 and a positive CK20 was not considered as accurate in diagnosis $(P>0.05)$ as in the other cases.

A combination of CK7 and CK20 has been previously reported to provide effective discrimination between carcinomas and is often used as part of a panel with other tissueor organ-specific IHC markers to establish the primary site of malignancy [12]. An algorithmic and probabilistic approach and the use of a detailed database have been recommended in previous reports for effective assessment [8]. In a recent study [12], it has been suggested that CK7+/CK20+ may represent the profile of urothelial carcinoma, pancreatic ductul adenocarcinoma, ovarian mucinous carcinoma, bladder adenocarcinoma, gastric adenocarcinoma or cholangiocarcinoma. Accordingly, the CK7+/CK20- phenotype may indicate lung adenocarcinoma, lung small cell carcinoma, breast carcinoma, ovarian serous carcinoma, endometrial adenocarcinoma, cholangiocarcinoma, thyroid carcinoma, mesothelioma, pancreatic carcinoma or gastric carcinoma. In the same setting, CK7-/CK20+ expression may refer to colorectal adenocarcinoma or Merckel cell carcinoma, while CK7-/CK20 - to prostate adenocarcinoma, renal cell carcinoma, hepatocellular carcinoma, squamous cell carcinoma, adrenal cortical carcinoma or gastric adenocarcinoma [12]. However, it should be mentioned that difficulties in discrimination often occur, as the cytokeratin phenotype may be altered in metastatic disease as compared to the primary tumor origin [8].

IHC determination of the site of origin of carcinomas is often achievable. When a metastasis is determined to be a carcinoma on the basis of screening immunostains, a panel of tissue- or organ-specific markers can be used in an attempt to suggest (or confirm) the tumor origin. How- 
ever, diverse tumor-or organ-specific ICH markers may also react with variable tumor types. In this setting, in case of metastatic tumors of unknown origin, the use of a panel of IHC markers is strongly suggested [12]. In our study we used such a IHC panel in 67 patients who had bone metastases from an unknown primary origin. However, in 32 of these patients (47\%) the tumor origin remained undetermined. An explanation to this may be the fact that metastatic tumors can change geno- or phenotype. Hence, different immunoprofiles of primary carcinomas and their metastasis may exist [15]. On the other hand, carcinomas of the unknown primary site represent, by themselves, a separate clinical entity that accounts for about $5 \%$ of all cancers [16].

The subset of patients in our study, where IHC analysis did not determinate the primary tumor site, were found to present a poorer prognosis. Diagnosis of a metastatic carcinoma of an unknown primary site has long been considered synonymous with poor prognosis, with a median overall survival time range from 6 to 13 months. Empiric chemotherapy is generally administered in these patients, while the benefit of different treatments studied compared with best supportive care is still unclear [17]. The expression of the determinate IHC phenotype in high grade tumors might correspond to a better differentiated appearance, with a better prognosis. Considering these facts, even if further studies with wider populations are required, patients with an undifferentiated phenotype may have a poorer prognosis.

\section{Conclusions}

CK7 and CK20 provide effective discrimination between carcinomas in bone metastasis specimens. They are often used as part of a panel with other tissue- or organ-specific IHC markers to establish the primary tumor site. A combination of these markers when examined in tissue of bone metastases may determine the origin of the primary tumor; however in some patients, primary malignancy will not be identified. The latter patients are expected to present a poorer prognosis.

\section{Acknowledgements}

This paper is dedicated to the memory of one of the coauthors, Andrea Pellacani, whose passionate dedication to increasing knowledge for the benefit of cancer patients deserves our acknowledgment.

Conflict of interest: none declared

\section{Costantino Errani, MD}

Department of Orthopaedics

University of Bologna

Istituto Ortopedico Rizzoli

Via Pupilli, 1, 140136, Bologna, Italy

Tel: +390516366000

e-mail: costantino.errani@ior.it

Received: 4 Jan 2017

Accepted: 16 Mar 2017

\section{References}

1. Piccioli A, Maccauro G, Spinelli MS et al. Bone metastases of unknown origin: epidemiology and principles of management. J Orthop Traumatol 2015; 16: 81-86.

2. Nottebaert M, Exner GU, von Hochstetter AR et al. Metastatic bone disease from occult carcinoma: a profile. Int Orthop 1989; 13: 119-123.

3. Katagiri $\mathrm{H}$, Takahashi $\mathrm{M}$, Inagaki J et al. Determining the site of the primary cancer in patients with skeletal metastasis of unknown origin: a retrospective study. Cancer 1999; 86: 533-537.

4. Allemani C, Weir HK, Carreira $\mathrm{H}$ et al. Global surveillance of cancer survival 1995-2009: analysis of individual data for 25,676,887 patients from 279 population-based registries in 67 countries (CONCORD-2). Lancet 2015; 385: 977-1010.

5. Shibata H, Kato S, Sekine I et al. Diagnosis and treatment of bone metastasis: comprehensive guideline of the Japanese Society of Medical Oncology, Japanese Orthopedic Association, Japanese Urological Association, and Japanese Society for Radiation Oncology. ESMO Open 2016; 1: e000037.

6. Varadhachary GR, Abbruzzese JL, Lenzi R. Diagnostic strategies for unknown primary cancer. Cancer 2004; 100: 1776-1785.

7. Chu P, Wu E, Weiss LM. Cytokeratin 7 and cytokeratin 20 expression in epithelial neoplasms: a survey of 435 cases. Mod Pathol 2000; 13: 962-972.

8. Tot T. Cytokeratins 20 and 7 as biomarkers: usefulness in discriminating primary from metastatic adenocarcinoma. Eur J Cancer 2002; 38: 758-763.

9. Landau MS, Kuan SF, Chiosea Set al. BRAF-mutated microsatellite stable colorectal carcinoma: an aggressive adenocarcinoma with reduced CDX2 and increased cytokeratin 7 immunohistochemical expression. Hum Pathol 2014; 45: 1704-1712.

10. Gyure KA, Morrison AL. Cytokeratin 7 and 20 expression in choroid plexus tumors: utility in differentiating these neoplasms from metastatic carcinomas. Mod Pathol 2000; 13: 638-643.

11. Kummar S, Fogarasi M, Canova A et al. Cytokeratin 7 and 20 staining for the diagnosis of lung and colorectal adenocarcinoma. Br J Cancer 2002; 86: 1884-1887.

12. Krishna M. Diagnosis of metastatic neoplasms: an immunohistochemical approach. Arch Pathol Lab Med 2010; 134: 207-215.

13. Blumenfeld W, Turi GK, Harrison G et al. Utility of cytokeratin 7 and 20 subset analysis as an aid in the identification of primary site of origin of malignancy in cytologic specimens. Diagn Cytopathol 1999; 20: 63-66.

14. Moll R, Franke WW, Schiller DL et al. The catalog of human cytokeratins: patterns of expression in normal epithelia, tumors and cultured cells. Cell 1982; 31: 11-24.

15. Kulka J, Szekely B, Lukacs LV et al. Comparison of predictive immunohistochemical marker expression of primary breast cancer and paired distant metastasis using surgical material: a practice-based study. J Histochem Cytochem 2016; 64: 256-267.

16. Hainsworth JD, Greco FA. Treatment of patients with cancer of an unknown primary site. NEngl J Med 1993; 329: 257-263.

17. Sporn JR, Greenberg BR. Empirical chemotherapy for adenocarcinoma of unknown primary tumor site. Semin Oncol 1993; 20: 261-267. 